\title{
Hyperinsulinemia and thyroid peroxidase antibody in Chinese patients with papillary thyroid cancer
}

\author{
Xiaoyan $\mathrm{Guo}^{1)}$ *, Xinyan Chen $^{2)}$ *, Ce Zhang ${ }^{3)}$, Jiahuan Zhang ${ }^{1)}$ and Chunyu Zhang ${ }^{1)}$ \\ 1) Department of Geriatrics, the Second Affiliated Hospital of Dalian Medical University, Dalian 116023, China \\ 2) Department of Osteoporosis, Affiliated Zhongshan Hospital of Dalian University, Dalian 116001, China \\ 3) Department of Pharmacy, the Second Affiliated Hospital of Dalian Medical University, Dalian 116023, China
}

\begin{abstract}
This study aimed to investigate if hyperinsulinemia and/or insulin resistance was correlated with the occurrence of papillary thyroid cancer (PTC) in a group of Chinese patients. 258 inpatients were included in the study. According to the postoperative pathology results, all subjects were divided into PTC $(n=153)$ and control groups (with benign thyroid nodules, $n=105$ ). Body mass index (BMI), fasting glucose, fasting insulin, homeostasis model assessment of insulin resistance (HOMA-IR), thyroid-stimulating hormone (TSH), FT4, FT3, thyroid peroxidase antibody (TPOAb), thyroglobulin antibody (TGAb), serum uric acid (UA), and lipid levels. Fasting insulin levels, HOMA-IR values, TPOAb levels, serum TSH levels, and serum uric acid levels in the PTC group were higher than those in the control group $(p<0.05)$. However, no significant differences in age, gender, BMI, history of hypertension, and the levels of fasting plasma glucose, FT3, FT4, TGAb, total cholesterol, triglyceride, low-density lipoprotein, and high-density lipoprotein were observed between the two groups $(p>$ 0.05). After the multiple logistic regression analysis, the occurrence of PTC was positively associated with fasting insulin (odds ratio $[\mathrm{OR}]=1.048,95 \%$ confidence interval $[\mathrm{CI}]: 1.003-1.096, p=0.037)$ and TPOAb levels $(\mathrm{OR}=1.001,95 \% \mathrm{CI}$ : $1.000-1.002, p=0.032)$. Moreover, TPOAb level was positively correlated with vague margin $(r=0.126, p=0.045)$ and negatively correlated with homogeneous echo $(r=-0.179, p=0.004)$. However, fasting insulin levels were not correlated with pathological characteristics of PTC. Hyperinsulinemia and higher TPOAb levels might be the risk factors of PTC, but not disease severity in Chinese patients.
\end{abstract}

Key words: Hyperinsulinemia, Insulin resistance, Papillary thyroid cancer, Risk factor, Thyroid peroxidase antibody

AS THE MOST COMMON ENDOCRINE MALIGNANCY, the incidence of thyroid cancer in the past few years is higher than that of any other malignant tumor [1, 2]. Differentiated thyroid cancer accounted for approximately $90 \%$ of all thyroid cancers, and papillary thyroid cancer (PTC) was the most common type of welldifferentiated thyroid cancer $(75 \%)$ [3, 4]. Epidemiological and clinical data that suggested unknown factors might cause the increasing incidence of thyroid cancer $[5,6]$. Insulin resistance and hyperglycemia are considered to be important risk factors of several types of cancer, such as colorectal, endometrial, lung, breast cancer, prostatic, and endometrial cancers [7-11]. Patients with insulin resistance also have a higher prevalence of thyroid nodules and bigger thyroid glands. A small cross-

Submitted Aug. 30, 2018; Accepted Apr. 17, 2019 as EJ18-0358 Released online in J-STAGE as advance publication May 22, 2019 Correspondence to: Chunyu Zhang, Department of Geriatrics, the Second Affiliated Hospital of Dalian Medical University, No. 467 Zhongshan Road, Shahekou District, Dalian 116023, China. E-mail: 17709876116@163.com

*These are co-first authors and contributed equally to this work. sectional study reported that increased prevalence of insulin resistance was present in patients with differentiated thyroid carcinomas [12]. A study from Sahin et al. showed PTC patients are more insulin resistant [13]. Recently, Bae et al. also demonstrated that hyperinsulinemia and/or insulin resistance may be associated with the development of PTC in Korean women [14]. In contrast, a study suggested that no difference in insulin resistance or insulin levels between thyroid cancer and benign thyroid nodules [15]. Although previous studies investigated the relationship between hyperinsulinemia or insulin resistance and thyroid cancer, the results were controversial. Therefore, further explorations on relationship between hyperinsulinemia and thyroid cancer are still needed.

Herein, we aimed to study the correlation between hyperinsulinemia and insulin resistance with the development of PTC by comparing the differences between PTC and benign thyroid nodules in a group of Chinese patients. In addition, associations between fasting insulin and TPOAb levels with pathological characteristics of PTC were assessed in this cohort. 


\section{Materials and Methods}

\section{Subjects}

Our study was performed at the Second Affiliated Hospital of Dalian Medical University between March 2016 and August 2016. Clinical data of 363 inpatients at the thyroid surgery department were retrospectively collected. Individuals with the following characteristics were excluded from the study: those with a history of other cancer except thyroid cancer $(n=3)$, diabetes mellitus $(n=28)$, other types of thyroid cancer (i.e., follicular, medullary, or anaplastic; $n=7$ ), history of thyroidectomy $(n=14)$, previous thyroid medication therapy at any time $(n=4)$, the subjects who only had cytology from ultrasound-guided fine-needle aspiration biopsies, not surgery $(n=31)$, severe liver insufficiency (ChildPugh score 10 to 15 ) and severe renal insufficiency (estimated glomerular filtration rate $<30 \mathrm{~mL} / \mathrm{min} \times 1.73 \mathrm{~m}^{2}$ ) $(n=10)$, or those who reject operation $(n=8)$. No one has a history of neck ionizing $\mathrm{I}^{131}$ therapy. Ultimately, 258 subjects who underwent thyroidectomy with postoperative pathology were included in this study. The patients were divided into PTC $(n=153)$ and control groups (with benign thyroid nodules, $n=105$ ) based on the postoperative pathological results. The study was approved by the local institutional ethics committee of the Second Affiliated Hospital in Dalian Medical University.

\section{Anthropometric measurements}

Body weights $(\mathrm{kg})$ and heights $(\mathrm{cm})$ were measured without shoes and/or cap before surgery. Body mass index (BMI) was expressed as weight per height squared $\left(\mathrm{kg} / \mathrm{m}^{2}\right)$. The anthropometric measurements were performed in triplicate.

\section{Biochemical evaluations}

Blood samples were collected between 8:00 and 9:00 in the morning, with the patients fasting for at least $8 \mathrm{~h}$ preoperatively. Serum samples obtained after centrifuge were stored at $-80^{\circ} \mathrm{C}$ to prepare for assaying. Then, fasting insulin was assayed in our clinical laboratory and was determined using chemiluminescence with antibodycoated tubes (ADVIA Centaur XP, chemiluminescence analyzer, Siemens, USA). The cutoff value of $>15$ $\mathrm{IU} / \mathrm{mL}$ was used to define hyperinsulinemia. Fasting plasma glucose level was measured using the glucose oxidase method (ADVIA 2400, automatic biochemical analyzer, Siemens, USA). The homeostasis model assessment of insulin resistance (HOMA-IR) level, an indicator of insulin resistance, was calculated with the formula fasting serum insulin $(\mathrm{IU} / \mathrm{mL}) \times$ fasting plasma glucose $(\mathrm{mmol} / \mathrm{L}) / 22.5$ [16]. A HOMA-IR index $\geq 2.5$ denoted insulin resistance [16]. Free T4, free T3, thyroid-stimulating hormone (TSH), thyroid peroxidase antibody (TPOAb), and thyroglobulin antibody (TGAb) levels were measured using chemiluminescence (ADVIA CentaurXP, chemiluminescence analyzer, Siemens, USA). Serum total cholesterol, triglyceride, and serum uric acid were measured using enzyme colorimetric method. Low-density lipoprotein cholesterol (LDL-C) and high-density lipoprotein cholesterol (HDL-C) were measured using direct method. The levels of serum uric acid, cholesterol, triglyceride, LDL-C, and HDL-C were determined using the ADVIA 2400 automatic biochemical analyzer (Siemens, USA). The above biochemical evaluations were performed in triplicate.

\section{Statistical analysis}

Data were analyzed using SPSS software (Statistical Package for the Social Sciences, version 22.0). The measurement data are presented as means \pm standard deviations (SDs) for continuous variable. Categorical variables are interpreted by frequency tables.

An independent sample $t$-test was used to compare distributed variables. On the other hand, the MannWhitney U-rank sum test was used for non-normally distributed variables. The Chi-square test was employed to analyze categorical variables when appropriate. $P$-values $<0.05$ were considered statistically significant. Logistic regression models were utilized to estimate odds ratios (ORs; 95\% confidence intervals [CIs]) to define the associations among fasting insulin, HOMA-IR, TSH and TPOAb with the prevalence of PTC. Additionally, a multivariate logistic regression analysis was performed to evaluate the associations of fasting insulin, HOMA-IR, TSH and TPOAb with the prevalence of PTC after adjusting for other three variables. To ensure that there were no collinearity problems, we implemented a collinearity diagnostic procedure, the variance inflation factor (VIF), to ensure low correlation (VIF lower than 10) between the variables in the multiple regression model. It was showed no collinearity among fasting insulin, HOMA-IR, TSH and TPOAb.

\section{Results}

\section{Clinical and metabolic characteristics of PTC and control groups}

Clinical and laboratory characteristics of PTC and control groups are shown in Table 1. No significant difference in age, BMI, gender, fasting plasma glucose levels, serum FT3 levels, serum FT4 levels, TGAb levels, total cholesterol levels, triglyceride levels, LDL levels, HDL levels, and history of hypertension (systolic pressure $\geq 140 \mathrm{mmHg}$ or diastolic pressure $\geq 90 \mathrm{mmHg}$ ) was 
Table 1 Clinical and metabolic characteristics of PTC group and control group

\begin{tabular}{lccc}
\hline Variables & $\begin{array}{c}\text { Control group } \\
(n=105)\end{array}$ & $\begin{array}{c}\text { PTC group } \\
(n=153)\end{array}$ & $p$-value \\
\hline Age $($ year $)$ & $48.42 \pm 11.85$ & $45.67 \pm 11.61$ & 0.065 \\
\hline Gender $($ female/male, $n)$ & $80 / 25$ & $116 / 37$ & 0.945 \\
\hline BMI $\left(\mathrm{kg} / \mathrm{m}^{2}\right)$ & $24.57 \pm 3.50$ & $25.13 \pm 3.94$ & 0.243 \\
\hline FPG $(\mathrm{mmol} / \mathrm{L})$ & $5.45 \pm 1.05$ & $5.41 \pm 0.75$ & 0.717 \\
\hline Fasting insulin $(\mathrm{uU} / \mathrm{mL})$ & $7.82 \pm 5.88$ & $10.00 \pm 8.14$ & 0.019 \\
\hline HOMA-IR & $1.94 \pm 1.81$ & $2.46 \pm 2.09$ & 0.042 \\
\hline TSH $(\mathrm{uIU} / \mathrm{mL})$ & $1.40(0.78,2.09)$ & $1.82(1.17,2.67)$ & 0.005 \\
\hline FT3 $(\mathrm{pmol} / \mathrm{L})$ & $4.78 \pm 0.70$ & $4.86 \pm 0.71$ & 0.082 \\
\hline FT4 $(\mathrm{pmol} / \mathrm{L})$ & $15.39 \pm 2.05$ & $15.54 \pm 2.66$ & 0.149 \\
\hline TPOAb $(\mathrm{U} / \mathrm{mL})$ & $28.0(9.29,489.64)$ & $31.3(10.05,1,301.00)$ & 0.015 \\
\hline TGAb $(\mathrm{U} / \mathrm{mL})$ & $25.6(10.5,501.00)$ & $28.8(12.30,393.31)$ & 0.511 \\
\hline Total cholesterol $(\mathrm{mmol} / \mathrm{L})$ & $4.82 \pm 0.77$ & $4.83 \pm 0.73$ & 0.893 \\
\hline Triglyceride $(\mathrm{mmol} / \mathrm{L})$ & $1.52 \pm 0.92$ & $1.53 \pm 0.92$ & 0.929 \\
\hline LDL $(\mathrm{mmol} / \mathrm{L})$ & $2.59 \pm 0.61$ & $2.60 \pm 0.58$ & 0.924 \\
\hline HDL $(\mathrm{mmol} / \mathrm{L})$ & $1.29 \pm 0.25$ & $1.27 \pm 0.32$ & 0.616 \\
\hline Serum uric acid $(\mathrm{umol} / \mathrm{L})$ & $287.42 \pm 76.89$ & $307.78 \pm 83.94$ & 0.049 \\
\hline Hypertension $(n, \%)$ & $13(12.4 \%)$ & $26(17.0 \%)$ & 0.310 \\
\hline PTC, papilary & & & \\
\hline
\end{tabular}

PTC, papillary thyroid cancer; BMI, body mass index; FPG, fasting plasma glucose; HOMA-IR, homeostasis model assessment of insulin resistance; TGAb, thyroglobulin antibody; TPOAb, thyroid peroxidase antibody; HDL, high-density lipoprotein; LDL, low-density lipoprotein.

Table 2 Logistic regression analysis showing the associations of fasting insulin, HOMA-IR, TSH and TPOAb with the prevalence of PTC

\begin{tabular}{lcclccc}
\hline & \multicolumn{2}{c}{ Univariate analysis } & & \multicolumn{2}{c}{ Multivariate analysis } \\
\cline { 2 - 3 } \cline { 6 - 7 } & OR & $95 \% \mathrm{CI}(p$ value $)$ & & OR & $95 \% \mathrm{CI}(p$ value $)$ \\
\hline Fasting insulin $(\mathrm{uU} / \mathrm{mL})$ & 0.951 & $0.909-0.994(0.027)$ & & 1.048 & $1.003-1.096(0.037)$ \\
HOMA-IR & 0.859 & $0.736-1.004(0.056)$ & & 1.535 & $0.832-2.832(0.171)$ \\
TSH $(\mathrm{uIU} / \mathrm{mL})$ & 1.016 & $0.958-1.078(0.593)$ & & 1.054 & $0.972-1.142(0.202)$ \\
TPOAb $(\mathrm{U} / \mathrm{mL})$ & 0.999 & $0.998-1.000(0.018)$ & & 1.001 & & $1.000-1.002(0.032)$ \\
\hline
\end{tabular}

observed between the PTC and control groups $(p>0.05)$. Fasting insulin levels, HOMA-IR values, TPOAb levels, serum TSH levels, and serum uric acid levels in the PTC group were significantly higher than those in the control group $(p<0.05)$.

\section{Logistic regression analysis on clinical and metabolic characteristics between the two groups}

In unadjusted logistic regression models, higher fasting insulin, HOMA-IR and TPOAb were strongly correlated with the occurrence of PTC (Table 2). As univariat analysis only considered the influence of single factor on PTC without considering the influence between factors, further multivariate analysis was performed.
In the multiple logistic regression analysis, PTC was associated with fasting insulin and TPOAb levels positively. The adjusted ORs (95\% CI) for subjects were 1.048 (95\% CI: $1.003-1.096, p=0.037)$ for fasting insulin levels and 1.001 (95\% CI: 1.000-1.002, $p=0.032$ ) for TPOAb (Table 2). However, there was no correlation between insulin level and TPO Ab titer $(r=0.067, p=$ $0.54)$.

\section{Associations between fasting insulin and TPOAb levels with pathological characteristics of PTC}

The pathology reports for PTC patients were reviewed to determine the severity of cancer as evidenced by the microcalcification (53 cases), streak-like calcifications 
Table 3 Associations among fasting insulin and TPOAb levels with pathological characteristics of PTC

\begin{tabular}{lcccccc}
\hline \multirow{2}{*}{ Pathological characteristics } & \multicolumn{2}{c}{ Fasting insulin } & & \multicolumn{2}{c}{ TPOAb } \\
\cline { 2 - 3 } \cline { 6 - 7 } & $r$ value & $p$ value & & $r$ value & $p$ value \\
\hline Microcalcification & -0.12 & 0.871 & & 0.033 & 0.600 \\
Streak-like calcifications & 0.095 & 0.201 & & 0.099 & 0.117 \\
Coarse calcification & -0.082 & 0.268 & & -0.086 & 0.171 \\
\hline Single nodule & 0.05 & 0.501 & & -0.036 & 0.571 \\
Multiple nodules & -0.065 & 0.381 & & 0.033 & 0.602 \\
Vague margin & 0.066 & 0.37 & & 0.126 & 0.045 \\
Nodule with a longitudinal/transverse ratio of $>1$ & -0.091 & 0.215 & & 0.051 & 0.417 \\
Blood flow & -0.027 & 0.718 & & 0.023 & 0.715 \\
\hline Homogeneous echo & 0.016 & 0.833 & & -0.179 & 0.004 \\
\hline Lymph node metastasis & 0.069 & 0.1352 & & 0.036 & 0.615 \\
\hline Tumor distant metastasis & -0.101 & 0.176 & & 0.110 & 0.124 \\
\hline
\end{tabular}

(54 cases), coarse calcification (33 cases), single nodule (60 cases), multiple nodules (198 cases), vague margin (119 cases), nodule with a longitudinal/transverse ratio of $>1$ ( 65 cases), blood flow ( 224 cases), homogeneous echo (66 cases), lymph node metastasis ( 71 cases), and tumor distant metastasis (2 cases). As shown in Table 3, single nodule, multiple nodules, nodule with a longitudinal/transverse ratio of $>1$, microcalcification, streak-like calcifications, coarse calcification, vague margin, blood flow, homogeneous echo, lymph node metastasis, and tumor distant metastasis were not significantly associated with fasting insulin level. However, TPOAb level was positively correlated with vague margin $(r=0.126, p=$ $0.045)$ and negatively correlated with homogeneous echo $(r=-0.179, p=0.004)$.

\section{Discussion}

In the last 20 years, the incidence of differentiated thyroid cancer, especially PTC, has continuously and dramatically increased [2, 17]. Some studies believed that the major cause of this increasing incidence was the result of enhanced detection of early stage tumors [17]. Others argued that a true increase was also occurring and the causes of this increase were unknown $[1,2]$. Some risk factors that might contribute to thyroid cancers were iodine intake status (low for follicular thyroid cancer), female, neck radiation, hereditary conditions, previous thyroid disease, and radioactive fallout from nuclear accidents [3, 18]. However, several recent studies attempted to identify the relationship of metabolic syndrome with the development of thyroid cancer $[13,19]$.

Hyperinsulinemia is considered to be a factor in the relationship between insulin resistance and cancer. It appears that hyperinsulinemia is secondary to obesityinduced insulin resistance. Rezzonico et al. [20] found that hyperinsulinemia could directly induce thyroid proliferation, which resulted in larger thyroid volume and the formation of thyroid nodules. A recent study from Bae et al. found that hyperinsulinemia was strongly associated with the risk of PTC and the multifocality of this disease [13]. In our study, we revealed that hyperinsulinemia was associated with the occurrence of PTC. These findings in our study were in accordance with previous studies $[13,20]$, further demonstrating that hyperinsulinemia might be the risk factor of PTC.

Hyperinsulinemia and insulin resistance are tightly linked each other. HOMA-IR, the mathematical product of fasting glucose and insulin, was independently associated with cancer mortality [21]. A previous literature showed patients with insulin resistance had larger thyroid volume and higher risk for formation of thyroid nodules [22]. Rezzonico et al. found that 20 patients with thyroid cancer exhibited increased HOMA-IR value than the control subjects [12]. Sahin et al. [14] showed that HOMA-IR value was high in patients with PTC in an iodine sufficient area. Recently, a study enrolled 735 Korean women showed that a higher HOMA-IR was significantly associated with the occurrence of PTC [13]. In contrast, Balkan et al. [15] did not find any difference in HOMA-IR index between differentiated thyroid cancer and benign thyroid nodules. The inconsistency of these results may be due to different pathological diagnosis. Some pathologic results were based on fine-needle aspiration cytology, which may deviate from the lesion in the lump, and some pathological results were based on tissues obtained after thyroidectomy. In our study, all patients underwent pathological analysis after thyroidec- 
tomy and we found that HOMA-IR value in the PTC group was significantly higher than that in the control group, however, HOMA-IR lost its statistical significance in multivariate model. It was reported that HOMA-IR can accurately describe insulin resistance in normal glucose tolerance population, while it should be cautious to use HOMA-IR in diabetic patients with abnormal glucose regulation, especially those with poor blood glucose control [23]. In our study, the number of undiagnosed diabetes in the included subjects is not clear, which may be a factor lead to no statistical differences on the association between HOMA-IR and PTC.

TPOAb and TGAb were produced by the thyroid gland. As a type of autoimmune thyroid disease, Hashimoto's thyroiditis had higher TGAb and TPOAb levels, which might promote the occurrence and development of thyroid cancer [19]. Higher TGAb and TPOAb levels might contribute to the development of thyroid cancer [24]. Previous studies showed that TGAb was a predictor for thyroid cancer, but not TPOAb [25, 26]. A study on palpable thyroid nodules found that TPOAb was associated with an increased risk of malignancy; however, this association was lost after adjusting for TSH [27]. Our study showed that higher TPOAb levels were a risk factor of PTC. Cipollini et al. [28] also reported that the occurrence of differentiated thyroid carcinoma was associated with TPOAb genetic variants. The mutation status of TPOAb gene was not analyzed in the present study. Whether stratified analysis of the mutation status of TPOAb gene will lead to different conclusions regarding the TPOAb role in PTC still needs further study.

TSH was an important hormone to promote the growth of thyroid cells and thyroid hormone synthesis. Boelaert et al. [27] reported that serum TSH concentration could be a predictor of malignancy in thyroid nodules through the fine-needle aspiration. A meta-analysis of 28 studies showed that the proportion of thyroid cancer in patients with thyroid nodules with decreased serum TSH levels was lower than those with normal or elevated serum TSH levels [29]. Recently, Tam et al. showed an association between serum TSH and aggressive variants of PTC regardless of autoimmunity [30]. All these findings suggest that TSH plays a vital role in thyroid cancer initiation and aggressiveness. In this study, we found that serum TSH levels in PTC patients were significantly higher than those in patients with benign thyroid nodules, which was consistent with these findings. In contrary to these findings, Kim et al. did not find higher TSH levels in advanced stage of differentiated thyroid cancer, which may be attributed to the imbalance between patients with benign disease (249 cases) and patients with PTC (1,080 cases) [31]. Another study also did not demonstrate association between serum TSH concentration and the prevalence of lymph node metastasis, extrathyroidal invasion, diffusion, and advanced stages in differentiated thyroid cancer patients [32]. Our multivariate model analysis did not found that serum TSH levels were associated with the prevalence of PTC. There may be some explanations for these results. The sample size in this study was small. In addition, the number of undiagnosed diabetes in the included subjects is not clear, which may be a factor lead to no statistical differences on the association between serum TSH and the prevalence of PTC.

This study has several limitations. First, this is a single-center study and the sample size was not large enough. Second, glucose clamp method is the gold standard in measuring insulin resistance. However, using it to estimate insulin resistance is unrealistic in our clinical practice. Third, this was a retrospective study; therefore, section or confounding bias may occur. Fourth, we only excluded the subjects with a history of diabetes and the number of undiagnosed diabetes in the included subjects is not clear. Therefore, analysis on the association between metabolic factors and the prevalence of PTC may be affected.

In conclusion, hyperinsulinemia and high TPOAb levels are the risk factors of PTC in China. However, as this study is a cross-sectional design and it was difficult to know their causal relationship with PTC. Therefore, additional longitudinal and observational studies with a large sample size are needed to further evaluate the effects of hyperinsulinemia and TPOAb level on the development and progression of PTC.

\section{Funding}

This study was funded by the Project of Dalian Science and Technology (grant number 2015E12SF152).

\section{Disclosure}

None of the authors have any potential conflicts of interest associated with this research.

\section{Ethical Approval}

All procedures performed in studies involving human participants were in accordance with the ethical standards of the institutional and/or national research committee and with the 1964 Helsinki declaration and its later amendments or comparable ethical standards. The study was approved by the local institutional ethics committee of the Second Affiliated Hospital in Dalian Medical University. 


\section{References}

1. Ito Y, Nikiforov YE, Schlumberger M, Vigneri R (2013) Increasing incidence of thyroid cancer: controversies explored. Nat Rev Endocrinol 9: 178-184.

2. Pellegriti G, Frasca F, Regalbuto C, Squatrito S, Vigneri R (2013) Worldwide increasing incidence of thyroid cancer: update on epidemiology and risk factors. $J$ Cancer Epidemiol 2013: 965212.

3. Cooper DS, Doherty GM, Haugen BR, Kloos RT, Lee SL, et al. (2009) Revised American Thyroid Association management guidelines for patients with thyroid nodules and differentiated thyroid cancer. Thyroid 19: 1167-1214.

4. Roy R, Kouniavsky G, Venkat R, Felger EA, Shiue Z, et al. (2012) The role of preoperative neck ultrasounds to assess lymph nodes in patients with suspicious or indeterminate thyroid nodules. J Surg Oncol 105: 601-605.

5. Enewold L, Zhu K, Ron E, Marrogi AJ, Stojadinovic A, et al. (2009) Rising thyroid cancer incidence in the United States by demographic and tumor characteristics, 1980 2005. Cancer Epidemiol Biomarkers Prev 18: 784-791.

6. Vigneri R, Malandrino P, Vigneri P (2015) The changing epidemiology of thyroid cancer: why is incidence increasing? Curr Opin Oncol 27: 1-7.

7. Soliman PT, Wu D, Tortolero-Luna G, Schmeler KM, Slomovitz BM, et al. (2006) Association between adiponectin, insulin resistance, and endometrial cancer. Cancer 106: 2376-2381.

8. Siddique A, Kowdley KV (2011) Insulin resistance and other metabolic risk factors in the pathogenesis of hepatocellular carcinoma. Clin Liver Dis 15: 281-296.

9. Djiogue S, Nwabo Kamdje AH, Vecchio L, Kipanyula MJ, Farahna M, et al. (2013) Insulin resistance and cancer: the role of insulin and IGFs. Endocr Relat Cancer 20: R1R17.

10. Pisani P (2008) Hyper-insulinaemia and cancer, metaanalyses of epidemiological studies. Arch Physiol Biochem 114: 63-70.

11. Hsing AW, Sakoda LC, Chua S Jr (2007) Obesity, metabolic syndrome, and prostate cancer. Am J Clin Nutr 86: s843-s857.

12. Rezzonico JN, Rezzonico M, Pusiol E, Pitoia F, Niepomniszcze H (2009) Increased prevalence of insulin resistance in patients with differentiated thyroid carcinoma. Metab Syndr Relat Disord 7: 375-380.

13. Sahin M, Ucan B, Ginis Z, Topaloglu O, Gungunes A, et al. (2013) Vitamin D3 levels and insulin resistance in papillary thyroid cancer patients. Med Oncol 30: 589.

14. Bae MJ, Kim SS, Kim WJ, Yi YS, Jeon YK, et al. (2016) High prevalence of papillary thyroid cancer in Korean women with insulin resistance. Head Neck 38: 66-71.

15. Balkan F, Onal ED, Usluogullari A, Tuzun D, Ozdemir D, et al. (2014) Is there any association between insulin resistance and thyroid cancer?: a case control study. Endocrine 45: 55-60.

16. Matthews DR, Hosker JP, Rudenski AS, Naylor BA, Treacher DF, et al. (1985) Homeostasis model assessment: insulin resistance and beta-cell function from fasting plasma glucose and insulin concentrations in man. Diabetologia 28: 412-419.

17. Guay B, Johnson-Obaseki S, McDonald JT, Connell C, Corsten M (2014) Incidence of differentiated thyroid cancer by socioeconomic status and urban residence: Canada 1991-2006. Thyroid 24: 552-555.

18. Belfiore A, La Rosa GL, La Porta GA, Giuffrida D, Milazzo G, et al. (1992) Cancer risk in patients with cold thyroid nodules: relevance of iodine intake, sex, age, and multinodularity. Am J Med 93: 363-369.

19. Konturek A, Barczynski M, Wierzchowski W, Stopa M, Nowak W (2013) Coexistence of papillary thyroid cancer with Hashimoto thyroiditis. Langenbecks Arch Surg 398: 389-394.

20. Rezzonico J, Rezzonico M, Pusiol E, Pitoia F, Niepomniszcze H (2008) Introducing the thyroid gland as another victim of the insulin resistance syndrome. Thyroid 18: 461-464.

21. Perseghin G, Calori G, Lattuada G, Ragogna F, Dugnani $\mathrm{E}$, et al. (2012) Insulin resistance/hyperinsulinemia and cancer mortality: the cremona study at the 15 th year of follow-up. Acta Diabetol 49: 421-428.

22. Ayturk S, Gursoy A, Kut A, Anil C, Nar A, et al. (2009) Metabolic syndrome and its components are associated with increased thyroid volume and nodule prevalence in a mild-to-moderate iodine-deficient area. Eur J Endocrinol 161: 599-605.

23. Insulin Resistance Group, Diabetes Society, Chinese Medical Association (2018) Expert guidance on insulin resistance assessment method and application. Chinese Journal of Diabetes 10: 377-385 (In Chinese).

24. Zhang MF, Yu HU, Zhou GY (2014) Association of serum antithyroid antibodies with risk of thyroid cancer. Chinese Journal of General Practice 12: 356-358 (In Chinese).

25. Kim ES, Lim DJ, Baek KH, Lee JM, Kim MK, et al. (2010) Thyroglobulin antibody is associated with increased cancer risk in thyroid nodules. Thyroid 20: 885891.

26. Vasileiadis I, Boutzios G, Charitoudis G, Koukoulioti E, Karatzas T (2014) Thyroglobulin antibodies could be a potential predictive marker for papillary thyroid carcinoma. Ann Surg Oncol 21: 2725-2732.

27. Boelaert K, Horacek J, Holder RL, Watkinson JC, Sheppard MC, et al. (2006) Serum thyrotropin concentration as a novel predictor of malignancy in thyroid nodules investigated by fine-needle aspiration. J Clin Endocrinol Metab 91: 4295-4301.

28. Cipollini M, Pastor S, Gemignani F, Castell J, Garritano S, et al. (2013) TPO genetic variants and risk of differentiated thyroid carcinoma in two European populations. Int $J$ Cancer 133: 2843-2851.

29. McLeod DS, Watters KF, Carpenter AD, Ladenson PW, Cooper DS, et al. (2012) Thyrotropin and thyroid cancer diagnosis: a systematic review and dose-response meta- 
analysis. J Clin Endocrinol Metab 97: 2682-2692.

30. Tam AA, Ozdemir D, Aydin C, Bestepe N, Ulusoy S, et al. (2018) Association between preoperative thyrotrophin and clinicopathological and aggressive features of papillary thyroid cancer. Endocrine 59: 1-8.

31. Kim HK, Yoon JH, Kim SJ, Cho JS, Kweon SS, et al.
(2013) Higher TSH level is a risk factor for differentiated thyroid cancer. Clin Endocrinol (Oxf) 78: 472-477.

32. He LZ, Zeng TS, Pu L, Pan SX, Xia WF, et al. (2016) Thyroid hormones, autoantibodies, ultrasonography, and clinical parameters for predicting thyroid cancer. Int $J$ Endocrinol 2016: 8215834. 